\title{
Thin-Layer Chromatography - Bioassay as Powerful Tool for Rapid Identification of Bioactive Components in Botanical Extracts
}

\author{
Snezana Agatonovic-Kustrin ${ }^{1 *}$, David W Morton ${ }^{1}$ and Ahmad P Yusof ${ }^{2}$ \\ ${ }^{1}$ School of Pharmacy and Applied Science, La Trobe Institute of Molecular Sciences, La Trobe University, Edwards Rd, Bendigo, 3550, Australia \\ ${ }^{2}$ Faculty of Medicine, Universiti Teknologi Mara, Sg. Buloh Campus, 47000, Sungai Buloh, Selangor, Malaysia
}

The plant kingdom, both terrestrial and marine, is an underestimated pool of secondary metabolites some of which have enormous therapeutic potential to be an important source of new and unique agents. The identification of active compounds from complex botanical extracts is the main challenge in natural products discovery. The strategy used to discover active substances has been considerably changed in the last decade from the expensive and laborious methods in which bioactivity was studied only after isolation and identification of the components. The new strategy involves only the effective components being isolated and identified. Since chemical separation of the whole sample does not provide information about biological effects, neither do bioassays of the whole sample extracts provide information on observed therapeutic effects for compounds found in these extracts. However, if chemical separation techniques are combined with bioassays the therapeutic effects of individual compounds in a plant extract are able to be assessed. The use of planar chromatography to first separate plant extracts into individual components followed by a bioassay offers a number of advantages. Many samples can be analyzed in parallel on the same chromatographic plate keeping analysis times short and costs low. The high flexibility in detection is accomplished with the use of various derivatization reagents. Hence, direct bioassay on the chromatographic plate makes Thin Layer Chromatography (TLC) screening a powerful tool for rapid identification of bioactive components in crude plant extracts. To search for bioactive compounds in plant extracts with a targeted activity, TLC is hyphenated with an appropriate bioassay enabling direct in vitro biological study of the components that have been previously separated on the plate. Hyphenation of TLC with bioassays, micro chemical detection and further Mass Spectroscopy (MS) identification enables targeted identification of substances from plant extracts. Post chromatographic derivatization by dipping or spraying can be done with either a universal micro chemical derivatization using for example anisaldehyde/sulfuric acid for detection of phenols, sugars, steroids, and terpenes [1] or with selective micro chemical derivatization. The diphenylpicrylhydrazyl $\left(\mathrm{DPPH}^{*}\right)$ free radical can be used to detect antioxidants or free radical scavengers [2] while fast blue salt $B$ is a stain that can used to detect various enzymes. Other reagents can be used to detect specific functional groups of phytochemical interest like natural products reagent (2-aminoethyl diphenylborinate) for flavonoids [2] or Brady's reagent (2,4-dinitrophenylhydrazine) for aldehydes and ketones [3].

For example the bioautographic procedure for screening for antimicrobial activity involves direct localization of antibacterial activity on a TLC chromatogram. The "agar diffusion" technique traditionally used, involves transfer of the antibacterial compound from the chromatographic plate to an inoculated agar plate by diffusion, where the zones of inhibition are then visualized. The bioautography technique is a simplified version of this method where a suspension of a microorganism in a suitable broth is directly applied to the TLC plate by dipping the plate into the broth [4]. The plate is than incubated in a humid atmosphere to allow growth of the bacteria and zones of inhibition are visualized after derivatization. Tetrazolium dyes, for example methylthiazolyltetrazlium bromide can be used to detect bacterial dehydrogenase activity. Yellow tetrazolium salts are reduced with bacterial dehydrogenases into the intensely colored bluish/violet formazans while antibacterial compounds (inhibition zones) will appear as colorless spots against a colored background.

A variety of in vitro methods can be used to test the antioxidant and free radical scavenging capacity of plant extracts [5]. These methods are based on the generation of free radical species which when added to a sample neutralize present antioxidants [6]. The observed decrease in free radical species concentration gives a measure of the antioxidant capacity of a sample. $\alpha, \alpha$-diphenyl- $\beta$-picrylhydrazyl $(\mathrm{DPPH})$ is a stable free radical that has a deep violet color in solution [7]. If a known amount of DPPH is added to a sample, the decrease in absorbance due to the disappearance of DPPH radical is directly proportional to the amount of antioxidants present in a sample [7]. However, the major disadvantage of this method is that it measures free radical scavenging ability of the whole extract, without being able to identify the most potent free radical scavengers in the sample [8]. Time consuming isolation of individual compounds can be avoided if the assay is directly run on a TLC plate. In the TLC-DPPH assay, a developed plate with the separated sample components, is sprayed or dipped in an alcohol DPPH free radical solution. Yellow spots present on the TLC plate against a purple background indicate the presence of an active antioxidant compound (Figure 1) [9].

Antioxidant activity of separated samples can be simply compared by superimposing the chromatograms of plant extracts obtained with TLC - DPPH assay methods against chromatograms of marker compounds (Figure 2). Free radical scavenging bands had $\mathrm{R}_{\mathrm{f}}$ values from 0.05 to 0.95 . Radical scavenging activity of the bands with $R_{f}$ values from 0.1-0.3 and bands with $\mathrm{R}_{\mathrm{f}}=0.75-0.85$ was highest, having more than half of the total scavenging activity. Assuming that structurally similar compounds have similar $\mathrm{R}_{\mathrm{f}}$ values it can be hypothesized that the most potent free radical scavengers in wine samples are polyphenolic nonflavonoids, i.e. resveratrol $\left(\mathrm{R}_{\mathrm{f}}=0.77 \pm 0.005\right)$ and flavonoids, like rutin $\left(\mathrm{R}_{\mathrm{f}}=0.18 \pm 0.005\right)$, while polyphenolic acids (caffeic acid and gallic acid) although present in higher concentrations in wines are less potent free radical scavengers [2]

Further combination of this system with spectroscopic and spectrometric techniques makes the characterization of the bioactive substances possible from their UV spectra by UV densitometric scanning $[10,11]$ and Direct Analysis in Real Time Mass Spectrometry (DART-MS) [12]. A mass spectrometric detector can be off-line

*Corresponding author: Snezana Agatonovic-Kustrin, School of Pharmacy and Applied Science, La Trobe Institute of Molecular Sciences, La Trobe University, Edwards Rd, Bendigo, 3550, Australia, Tel: +613-5444 7360; Fax: +613-54447878; E-mail: s.kustrin@latrobe.edu.au

Received January 07, 2015; Accepted January 08, 2015; Published January 12 2015

Citation: Agatonovic-Kustrin S, Morton DW, Yusof AP (2015) Thin-Laye Chromatography - Bioassay as Powerful Tool for Rapid Identification of Bioactive Components in Botanical Extracts. Mod Chem appl 3: e120. doi:10.4172/23296798.1000e120

Copyright: (c) 2015 Agatonovic-Kustrin S, et al. This is an open-access article distributed under the terms of the Creative Commons Attribution License, which permits unrestricted use, distribution, and reproduction in any medium, provided the original author and source are credited. 
Citation: Agatonovic-Kustrin S, Morton DW, Yusof AP (2015) Thin-Layer Chromatography - Bioassay as Powerful Tool for Rapid Identification of Bioactive Components in Botanical Extracts. Mod Chem appl 3: e120. doi:10.4172/2329-6798.1000e120
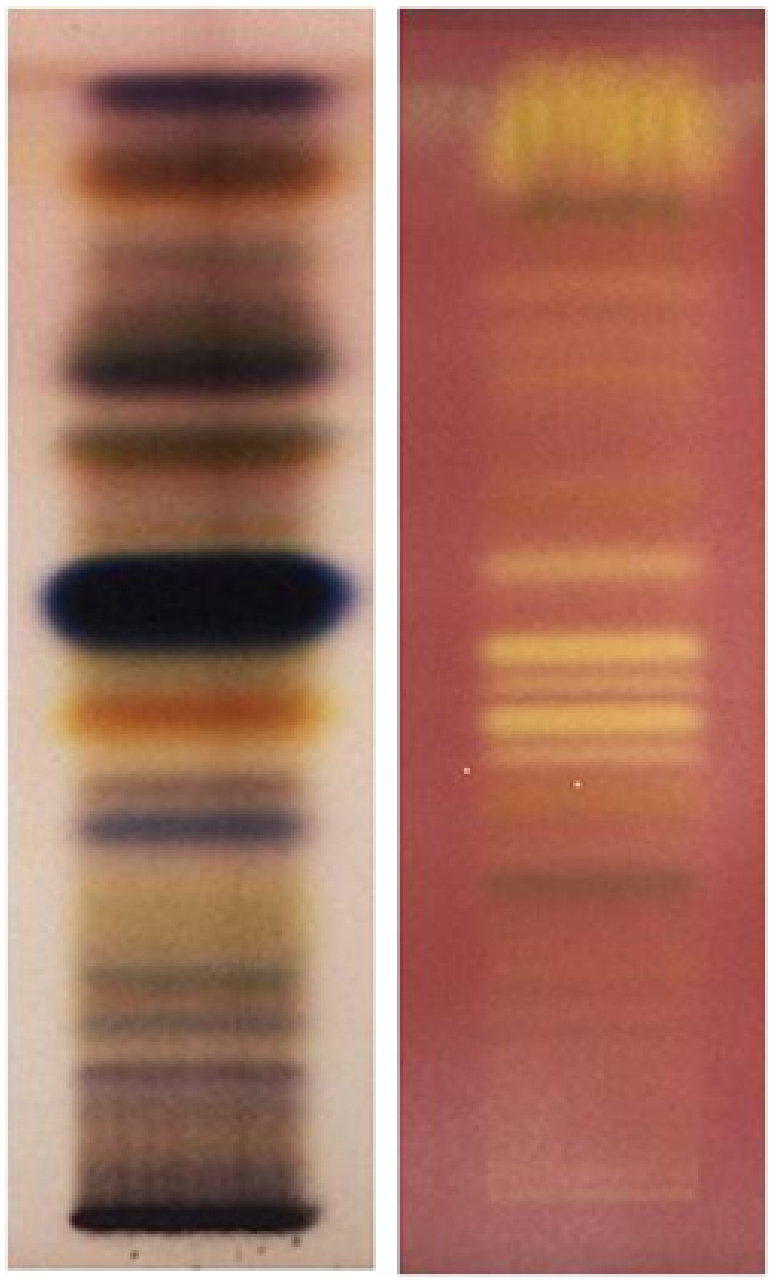

Figure 1: Feverfew extract separated on a TLC plate and derivatized with anisaldehyde (left) or derivatized with DPPH• (right).

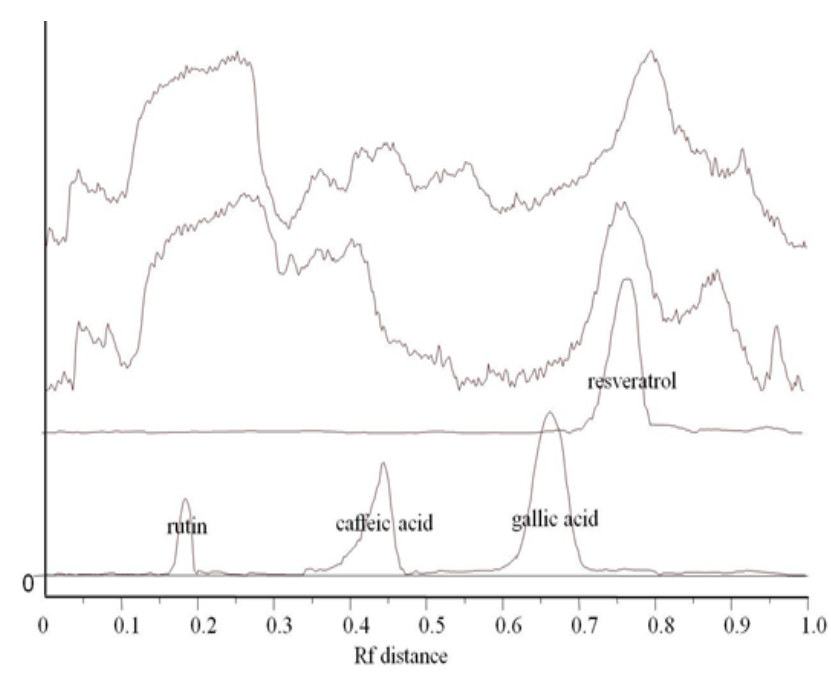

Figure 2: Superimposed chromatograms of 2 red wine samples derivatized with DPPH (two top chromatograms), and the chromatogram of the resveratrol standard (without derivatization) and rutin, caffeic acid and gallic acid after derivatization with Natural reagent (bottom chromatogram). coupled with the thin-layer chromatographic plate using a TLC-MS interface to characterize the active compounds [13]. Therefore, TLC chromatographic separation gains an additional analytical dimension with flexible separation and better identification than can be obtained using other methods.

\section{Conclusion}

TLC separation combined with direct bioassay enables highthroughput screening and characterization of the bioactive compounds from complex samples. These systems provide cheaper and less laborious ways to isolate substances with targeted biological activities.

\section{References}

1. Borhade VB, Nair HA, Hegde DD, Barhate CR (2009) Development and validation of HPTLC method for estimation of tacrolimus in formulations. Drug Dev Ind Pharm 35: 440-448.

2. Agatonovic-Kustrin S, Hettiarachchi CG, Morton DW, Razic S (2015) Analysis of phenolics in wine by high performance thin-layer chromatography with gradient elution and high resolution plate imaging. J Pharm Biomed Anal 102 93-99.

3. Ruekberg B, Rossoni E (2005) An Improved Preparation of 2,4-Dinitrophenylhydrazine Reagent. J Chem Educ 82: 1310.

4. Dewanjee S, Gangopadhyay M, Bhattacharya N, Khanra R, Dua TK (2014) Bioautography and its scope in the field of natural product chemistry. Journal of Pharmaceutical Analysis.

5. Arnao MB, Cano A, Acosta M (2001) The hydrophilic and lipophilic contribution to total antioxidant activity. Food Chem 73: 239-244.

6. Wozniak D, Janda B, Kapusta I, Oleszek W, Matkowski A (2010) Antimutagenic and anti-oxidant activities of isoflavonoids from Belamcanda chinensis (L.) DC. Mutat Res 696: 148-153.

7. Huang D, Ou B, Prior RL (2005) The chemistry behind antioxidant capacity assays. J Agric Food Chem 53: 1841-1856.

8. Kedare SB, Singh RP (2011) Genesis and development of DPPH method of antioxidant assay. J Food Sci Technol 48: 412-422.

9. Agatonovic-Kustrin S, Morton D, Yusof P (2012) Reversed Phase HPTLC DPPH Free Radical Assay as a Screening Method for Antioxidant Activity in Marine Crude Extracts. Oceanography 2: e112.

10. Venkateswarlu K, Venisetty RK, Yellu NR, Keshetty S, Pai MG (2007) Development of HPTLC-UV absorption densitometry method for the analysis of alprazolam and sertraline in combination and its application in the evaluation of marketed preparations. J Chromatogr Sci 45: 537-539.

11. Alam P (2013) Densitometric HPTLC analysis of 8-gingerol in Zingiber officinale extract and ginger-containing dietary supplements, teas and commercial creams. Asian Pac J Trop Biomed 3: 634-638.

12. Fukuda E, Uesawa Y, Baba M, Suzuki R, Fukuda T, et al. (2014) Identification of the country of growth of Sophora flavescens using direct analysis in real time mass spectrometry (DART-MS). Nat Prod Commun 9: 1591-1594.

13. Sajewicz M, Staszek D, Natic M, Waksmundzka-Hajnos M, Kowalska T (2011) TLC-MS versus TLC-LC-MS fingerprints of herbal extracts. Part III. Application of the reversed-phase liquid chromatography systems with $\mathrm{C} 18$ stationary phase. J Chromatogr Sci 49: 560-567. 